\title{
Scattering of linear and nonlinear waves in a waveguide array with a $\mathcal{P} \mathcal{T}$-symmetric defect
}

\author{
Sergey V. Dmitriev, ${ }^{1}$ Sergey V. Suchkov, ${ }^{1}$ Andrey A. Sukhorukov, ${ }^{2}$ and Yuri S. Kivshar ${ }^{2}$ \\ ${ }^{1}$ Institute for Metals Superplasticity Problems, Russian Academy of Science, Ufa RU-450001, Russia \\ ${ }^{2}$ Nonlinear Physics Centre, Research School of Physics and Engineering, Australian National University, Canberra, ACT 0200, Australia
}

(Received 24 May 2011; published 28 July 2011)

\begin{abstract}
We study the scattering of linear and nonlinear waves in a long waveguide array with a parity-time $(\mathcal{P} \mathcal{T})$ symmetric defect created by two waveguides with balanced gain and loss. We present exact solutions for the scattering of linear waves on such a defect, and then demonstrate numerically that the linear theory can describe, with a good accuracy, the soliton scattering in the case of weak nonlinearity. We reveal that the reflected and transmitted linear and nonlinear waves can be amplified substantially after interaction with the $\mathcal{P} \mathcal{T}$-symmetric defect thus allowing an active control of the wave scattering in the array.
\end{abstract}

DOI: 10.1103/PhysRevA.84.013833

PACS number(s): 42.81.Dp, 42.82.Et, 42.81.Qb, 42.25.Bs

\section{INTRODUCTION AND MODEL}

Photonic structures composed of coupled waveguides with loss and gain regions offer new possibilities for shaping optical beams and pulses compared to conservative structures [1-4]. Such structures can be designed as optical analogues of complex parity-time $(\mathcal{P} T)$-symmetric potentials, which can have a real spectrum corresponding to the conservation of power for optical eigenmodes, however the beam dynamics can demonstrate unique features distinct from conservative systems due to nontrivial wave interference and phase transition effects [5-10]. Most recently, $\mathcal{P} T$-symmetric properties in couplers composed of two waveguides were demonstrated experimentally $[11,12]$. Various schemes have been suggested to tailor beam shaping and switching using $\mathcal{P} T$-symmetric structures, including introduction of fabricated defects in periodic lattices [13] and employing self-induced refractive index change in nonlinear structures [1,14-18].

In this paper, we suggest and analyze nonlinear $\mathcal{P} \mathcal{T}$ symmetric systems, where a pair of waveguides with balanced gain and loss are embedded into an array of lossless waveguides, as illustrated schematically in Fig. 1(a). Since the active region is confined at only two waveguides, it can be anticipated that the experimental realization of such a structure can be simpler compared to the previously considered cases of nonlinear structures with a periodic arrangement of gain and loss elements [14-16]. On the other hand, the proposed structure offers a full potential for nonlinear switching and control combined with amplification. Specifically, we reveal that reflected and transmitted discrete soliton (self-localized beam supported by nonlinear conservative lattice) [19] can be substantially amplified by the $\mathcal{P} \mathcal{T}$-symmetric defect. We employ the linear scattering theory to explain this effect analytically.

We use the coupled-model equations $[10,12,16]$ to model the beam propagation in a nonlinear waveguide array with a local inhomogeneity created by a pair of $\mathcal{P} \mathcal{T}$-symmetric waveguides with balanced gain and loss [see Fig. 1(a)]:

$$
\begin{gathered}
i \frac{d a_{j}}{d z}+C_{1} a_{j-1}+C_{1} a_{j+1}+\gamma\left|a_{j}\right|^{2} a_{j}=0, j \neq 0,1, \\
i \frac{d a_{0}}{d z}+i \rho a_{0}+C_{1} a_{-1}+C_{2} a_{1}+\gamma\left|a_{0}\right|^{2} a_{0}=0 \\
i \frac{d a_{1}}{d z}-i \rho a_{1}+C_{2} a_{0}+C_{1} a_{2}+\gamma\left|a_{1}\right|^{2} a_{1}=0
\end{gathered}
$$

where $j$ is the waveguide number, $z$ is the propagation distance, $a_{j}$ are the mode amplitudes at waveguides, $\rho>0(<0)$ defines the rate of loss (gain) at zeroth and gain (loss) at first waveguides, $\gamma$ is the Kerr nonlinearity coefficient, and $C_{1,2}$ are the coupling coefficients between the modes of waveguides that can be tuned by changing the distance between the waveguides.

The paper is organized as follows. In Sec. II we analyze the scattering of linear waves by the composite $\mathcal{P} \mathcal{T}$-symmetric defect setting $\gamma=0$ in Eqs. (1)-(3), and derive some exact relations for the transmission and reflection coefficients. Then, in Sec. III we study numerically the soliton scattering for the case of weak nonlinearity $\left(\left|a_{j}\right|^{2} \ll 1\right)$. Finally, Sec. IV concludes the paper.

\section{LINEAR WAVE SCATTERING}

In the case of $\gamma=0$, which is the subject of this section, the model described by Eqs. (1)-(3) becomes linear, and it can be treated analytically.

\section{A. Transmission and reflection coefficients}

For $C_{1}=C_{2}$ and $\rho=0$, the waveguide array supports the eigenmode solutions in the form of Floquet-Bloch modes, $a_{j}(z)=A \exp (i k j-i \beta z)$, where $A$ is amplitude, $k$ is Bloch wave number, and $\beta$ is propagation constant obeying the following diffraction relation:

$$
\beta=-2 C_{1} \cos (k) .
$$

In a general case, the presence of gain and loss at zeroth and first waveguides and the difference of the coupling constant between these two waveguides from the other ones play the role of scatterer of the linear waves. In order to calculate the transmission and reflection coefficients we consider solution to Eqs. (1)-(3) of the form

$$
\begin{aligned}
& a_{j}=\exp (i k j-i \beta z)+R(\rho) \exp (-i k j-i \beta z), j \leqslant 0, \\
& a_{j}=T(\rho) \exp (i k j-i \beta z), j \geqslant 1,
\end{aligned}
$$

where the first line represents the incident and the reflected waves, and the second one-the transmitted wave. Obviously, Eq. (1) is satisfied automatically. Substituting Eq. (5) into 

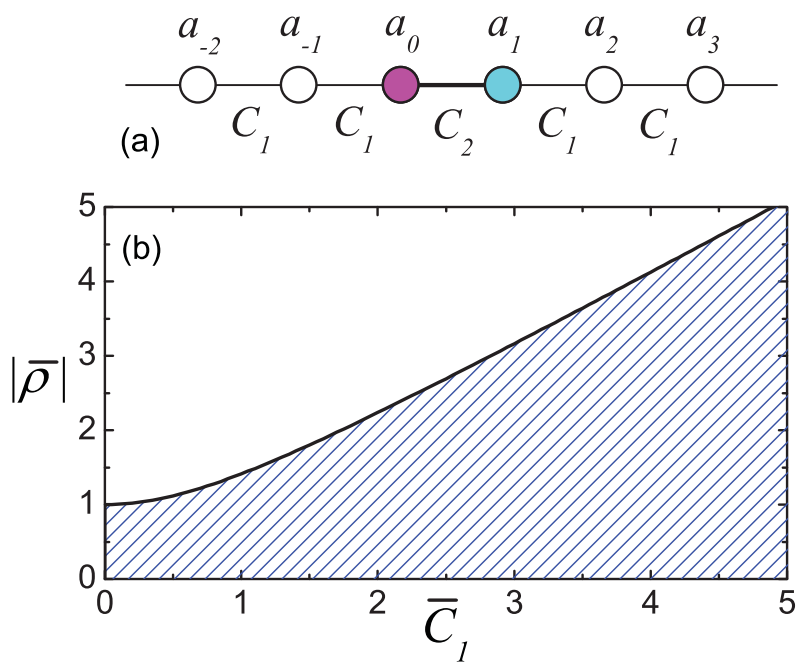

FIG. 1. (Color online) (a) Schematic of a waveguide array with a pair of $\mathcal{P} \mathcal{T}$-symmetric waveguides at sites $j=0,1$ with balanced gain and loss. Here $j$ is the waveguide number, $z$ is the propagation distance, $a_{j}$ are the mode amplitudes at waveguides, and $C_{1,2}$ are the coupling coefficients between the modes of waveguides. (b) Range of parameters (shaded) corresponding to the $\mathcal{P} \mathcal{T}$-symmetric regime where the power of all linear eigenmodes are conserved.

Eqs. (2) and (3) one finds the complex reflection and transmission coefficients

$$
\begin{aligned}
& T(\rho)=\frac{e^{-i k} 2 i \bar{C}_{1} \sin k}{D} \\
& R(\rho)=\frac{1-\bar{C}_{1}^{2}-\bar{\rho}^{2}+2 \bar{\rho} \bar{C}_{1} \sin k}{D},
\end{aligned}
$$

where

$$
\begin{aligned}
& D=\bar{C}_{1}^{2} \exp (-2 i k)+\bar{\rho}^{2}-1, \\
& \bar{\rho}=\frac{\rho}{C_{2}}, \quad \bar{C}_{1}=\frac{C_{1}}{C_{2}} .
\end{aligned}
$$

Note that $T(\rho)=T(-\rho)$ but $R(\rho) \neq R(-\rho)$. This implies that the intensity of the transmitted wave does not depend on whether the incident wave hits the waveguide with gain or with loss, while the intensity of the reflected wave does.

It can be easily demonstrated that $T$ and $R$ diverge for $k=\pi / 2$ for the model parameters satisfying

$$
|\bar{\rho}|=\left(1+\bar{C}_{1}^{2}\right)^{1 / 2} \equiv \bar{\rho}_{\text {crit }} .
$$

Stability analysis demonstrates that the waveguide array with a double $\mathcal{P} T$-symmetric defect is unstable with respect to small-amplitude waves for $|\bar{\rho}| \geqslant \bar{\rho}_{\text {crit }}$. The range of admissible model parameters is shaded in Fig. 1(b).

\section{B. Localized defect modes}

For $C_{1}<C_{2}$, i.e., $\bar{C}_{1}<1$, considered system Eqs. (1)-(3) supports the two localized eigenmodes

$$
\begin{aligned}
& a_{j}(z)=L q^{1-j} \exp (-i \beta z), \quad j \leqslant 0, \\
& a_{j}(z)=L q^{j} \exp (-i \beta z+i \varphi), \quad j \geqslant 1,
\end{aligned}
$$

with

$$
\begin{aligned}
\beta_{1,2} & = \pm\left(\bar{\rho}^{2}-\bar{C}_{1}^{2}-1\right)(1-\bar{\rho})^{-1 / 2}, \\
q_{2} & =\frac{1}{q_{1}}=-\frac{\beta_{1,2}}{2 C_{1}}+\left(\frac{\beta_{1,2}^{2}}{4 C_{1}^{2}}-1\right)^{1 / 2}, \\
\varphi_{1} & =-\sin ^{-1}(\bar{\rho}), \quad \varphi_{2}=\pi+\sin ^{-1}(\bar{\rho}),
\end{aligned}
$$

where $L$ is amplitude and $\beta$ is the mode propagation constant. For $\beta<0(>0)$ one has nonstaggered (staggered) mode with $q>0(<0)$. The propagation mode is real, i.e., the linear mode is a stationary state if $\left|C_{2}\right|>|\rho|$. For $C_{2}<C_{1}$ the localized mode cannot exist because its frequency lies within the frequency band of linear waves given by Eq. (4).

\section{Illustrations}

We now illustrate the analytical results with some numerical examples. In Fig. 2, for the transmission and reflection coefficients defined by Eq. (6), we plot $|R|^{2}$ (dotted line) and $|T|^{2}$ (solid line) as the functions of $k$ for different values of $\bar{\rho} / \bar{\rho}_{\text {crit }}$. Left column gives the results for $\bar{C}_{1}=1 / 4$ (localized mode can be excited) and the right one for $\bar{C}_{1}=4$ (localized mode cannot be excited).

As one can see from Fig. 2, intensity of the transmitted and reflected waves can be amplified significantly. Waves with $k$ close to $\pi / 2$ are amplified more intensively, and for $|\bar{\rho}|=\bar{\rho}_{\text {crit }}$,

(a)
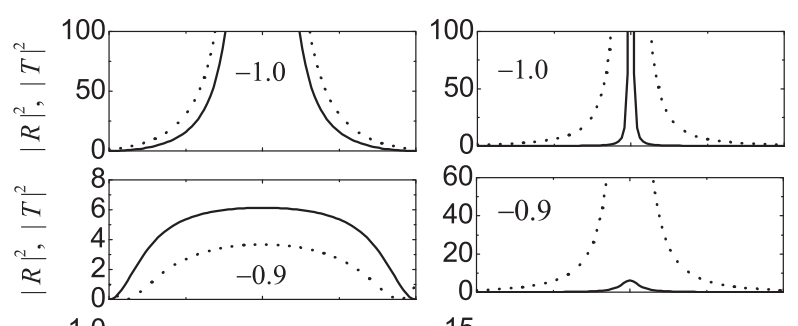

(c)
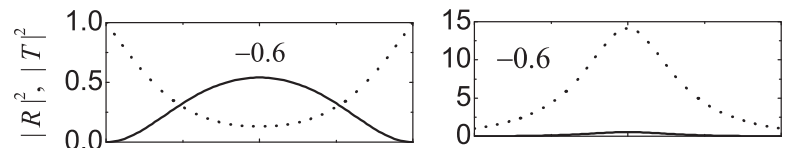

(d)
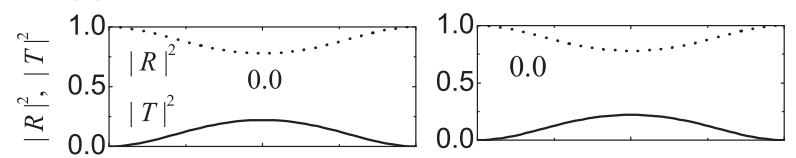

(e)

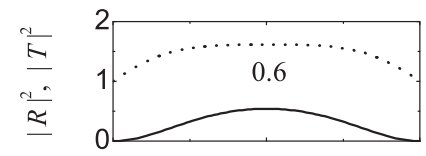

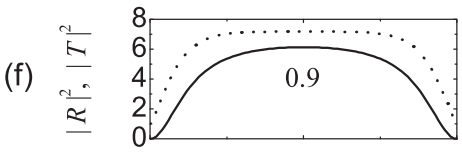

(g)
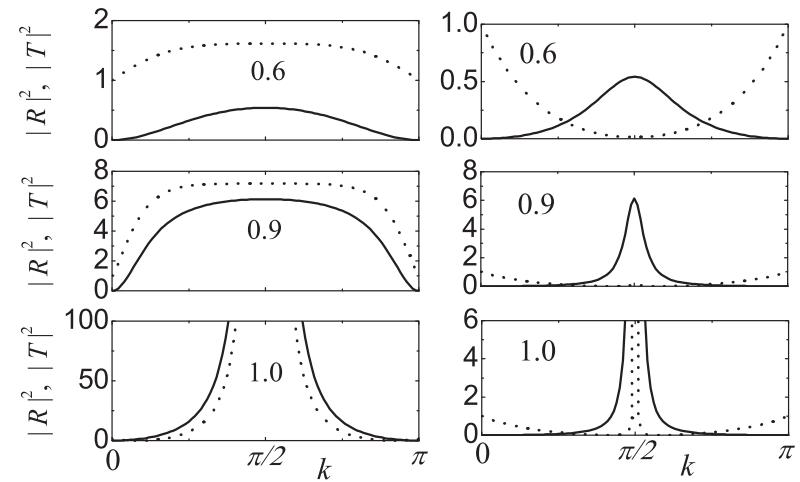

FIG. 2. Reflection $|R|^{2}$ (dotted line) and transmission $|T|^{2}$ (solid line) coefficients as the functions of $k$ for different values of $\bar{\rho} / \bar{\rho}_{\text {crit }}$ : (a) -1 , (b) -0.9 , (c) -0.6 , (d) 0, (e) 0.6, (f) 0.9, (g) 1. Left column: $\bar{C}_{1}=1 / 4$, right column: $\bar{C}_{1}=4$. 

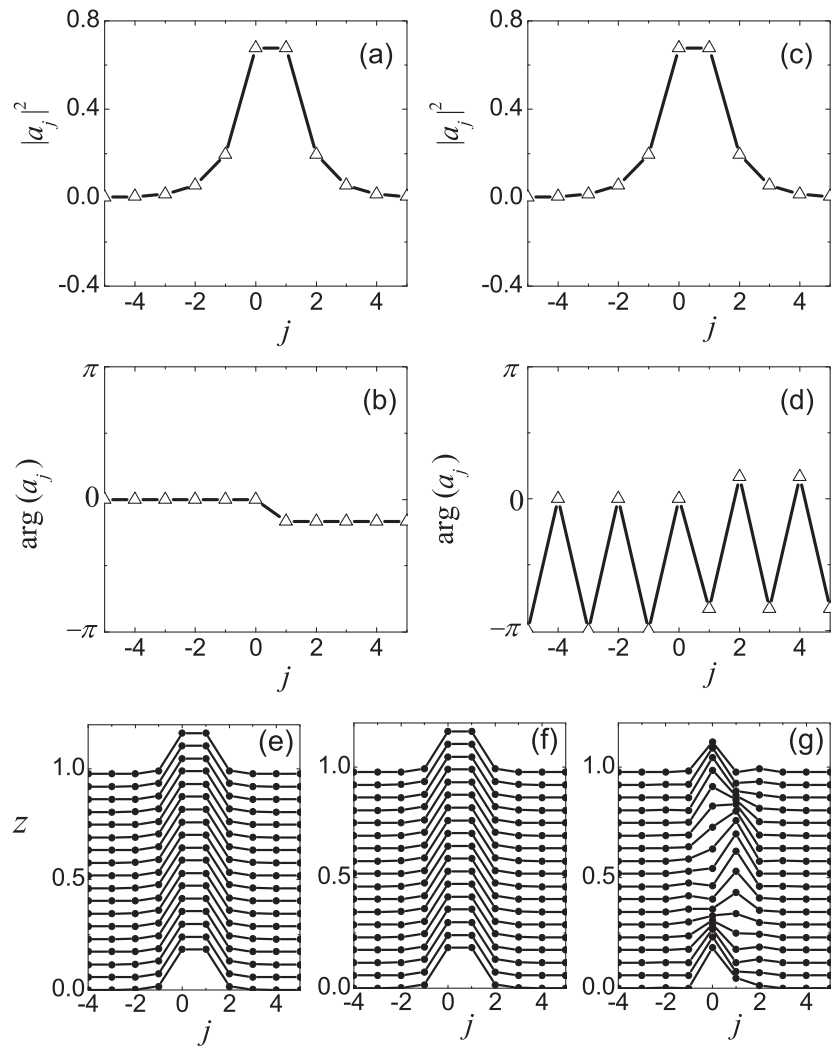

FIG. 3. (a,c) Intensity and (b,d) phase profiles of the localized modes given by Eqs. (9) and (10) for $C_{1}=1, C_{2}=4, \rho=2$ with (a,b) $\beta<0$ and (c,d) $\beta>0$. (e,f) 3D plots showing evolution of $\left|a_{j}(z)\right|^{2}$ for the localized mode presented in $(\mathrm{a}, \mathrm{c})$ and $(\mathrm{b}, \mathrm{d})$, respectively. (g) 3D plot for the linear combination of localized modes presented in $(\mathrm{a}, \mathrm{c})$ and $(\mathrm{b}, \mathrm{d})$ with equal amplitudes.

as it was already mentioned, the transmission and reflection coefficients diverge for waves with $k=\pi / 2$ [see Fig. 2(a) and $(\mathrm{g})]$. Note that in the case when the localized defect mode cannot be excited (left column in Fig. 2), transmitted and reflected waves can be amplified with almost constant amplification factor for a wide range of $k$ [see Fig. 2(b) and (f)].

In Fig. 3(a,b,e) we plot the nonstaggered localized mode given by Eqs. (9) and (10) with $\beta<0$ for $C_{1}=1, C_{2}=4$, $\rho=2$. In (a) the intensity profile, $\left|a_{j}\right|^{2}$, and in (b) the phase profile, $\arg \left(a_{j}\right)$, are presented. In (c), the three-dimensional (3D) plot showing the evolution of $\left|a_{j}(z)\right|^{2}$ for the localized mode depicted in $(a, b)$ is shown. In $(c, d, f)$ we present the same as in (a,b,e), respectively, but for the staggered mode with $\beta>$ 0 . Panel $(\mathrm{g})$ shows the linear combination of localized defect modes presented in (a,b,e) and (c,d,f) with equal amplitudes. In (e) the intensities of the waveguides with loss $(j=0)$ and gain $(j=1)$ periodically change from exactly zero to a maximal value, oscillating out-of-phase.

In Fig. 4, we show the scattering of the Gaussian beam,

$$
a_{j}(z=0)=\exp \left[i k\left(j-j_{0}\right)-\delta\left(j-j_{0}\right)^{2}\right],
$$

for the pulse parameters $\delta=0.01, k=\pi / 2$ (the case of zero second-order diffraction), $j_{0}=-50$, and model parameters $C_{1}=1, C_{2}=4$, and $\bar{\rho} / \bar{\rho}_{\text {crit }}=-0.9$. One can see that the reflected and transmitted pulses have amplitude considerably

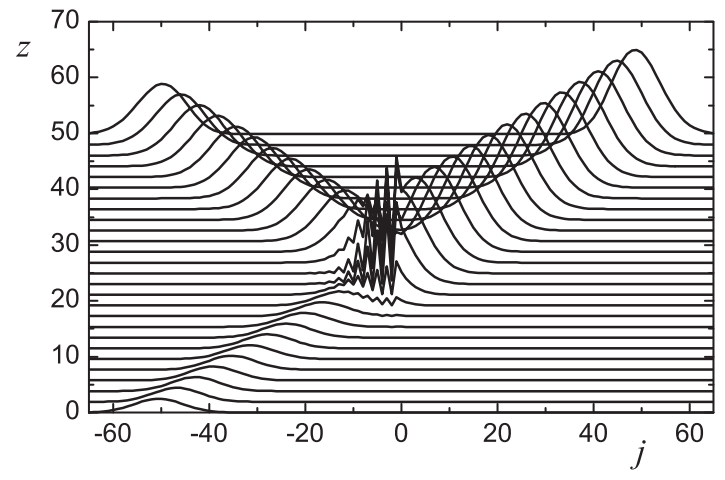

FIG. 4. 3D plot showing evolution of $\left|a_{j}(z)\right|^{2}$ for the scattering of the nondiffracting Gaussian pulse Eq. (11) with parameters $\delta=$ $0.01, k=\pi / 2, j_{0}=-50$. Model parameters are $C_{1}=1, C_{2}=4$, and $\bar{\rho} / \bar{\rho}_{\text {crit }}=-0.9$.

higher than the incident one. The amplification coefficients are equal to those corresponding to extended wave with $k=\pi / 2$. Model parameters in this example are the same as in the case shown in Fig. 2(b), where for $k=\pi / 2$ we have $|T|^{2}=6.13$ and $|R|^{2}=3.67$.

\section{SOLITON SCATTERING}

The Gaussian beam (11) does not preserve its shape with propagation in $z$ for $k \neq \pm \pi / 2$ due to the wave diffraction. On the other hand, it is well known that the nonlinearity effect can compensate for diffraction leading to the stable propagation of solitary waves. In this section, we study the interaction of solitary waves with $\mathcal{P} \mathcal{T}$-symmetric defect for the case of $\gamma>0$.

Far from the location of the defect, i.e., for $|j| \gg 1$, Eq. (1) has an approximate solution for a self-localized beam, where such solitons can propagate through the waveguide array $[19,20]$,

$$
a_{j}=A \operatorname{sech}\left[\delta\left(j-j_{0}-2 C_{1} v z\right)\right] e^{i\left[v\left(j-j_{0}\right)+\left(\delta^{2}-v^{2}\right) C_{1} z+\alpha\right]},
$$

which can be found by considering the continuum limit of Eq. (1) in the form of the integrable nonlinear Schrödinger equation [20]. Here $A, \delta=A \sqrt{\gamma /\left(2 C_{1}\right)}, v, j_{0}$, and $\alpha$ are parameters defining the soliton amplitude, inverse width, velocity, initial position, and initial phase, respectively. Whereas solitons moving in optical lattices may emit radiation [20], the radiative losses can be neglected for small propagation velocities.

In order to characterize the soliton scattering by a $\mathcal{P} \mathcal{T}$ symmetric defect, we define the soliton power as

$$
P=\sum_{j=-\infty}^{\infty}\left|a_{j}\right|^{2},
$$

where in practice the summation over $j$ is done around the soliton center to obtain $P$ with a desirable accuracy. We consider the incident soliton approaching the twowaveguide defect from the left, and calculate the relative power of the transmitted and reflected waves defined as follows:

$$
N_{T}=P_{T} / P_{I}, \quad N_{R}=P_{R} / P_{I},
$$


where $P_{I}, P_{T}$, and $P_{R}$ are the powers of the incident, transmitted, and reflected solitons, respectively. We calculate the powers $P_{T}$ and $P_{R}$, and also the amount of total power captured by the scatterer, after the transmitted and reflected solitons move well apart.

It is useful to compare the results of the soliton scattering with the prediction of the linear scattering theory. In this case, the relative intensities of the reflected and transmitted linear waves are denoted as

$$
\hat{N}_{T}=|T|^{2}, \quad \hat{N}_{R}=|R|^{2},
$$

where $R$ and $T$ are given by Eq. (6).

We aim to compare the numerically found relative powers of the reflected and transmitted solitons, Eq. (14), with that of the linear waves, Eqs. (6) and (15). We note that for a broad soliton beam, its spatial Fourier spectrum shall be centered around the wave number $k=v$, and we use this wave number value for comparison of linear and nonlinear scattering regimes.

For the model parameters, we always take $\gamma=1, C_{1,2}$ of order of unity, and rather small incident soliton amplitude, $A=0.05$, to make the soliton width much greater than the distance between nearest waveguides, when the approximate solution in Eq. (12) has high accuracy. Also for small soliton amplitude, $A \ll 1$, influence of the cubic term in Eqs. (1)-(3) is small and one has the regime of weak nonlinearity. We find that the soliton initial phase $\alpha$ has no effect on the scattering process and set $\alpha=0$.
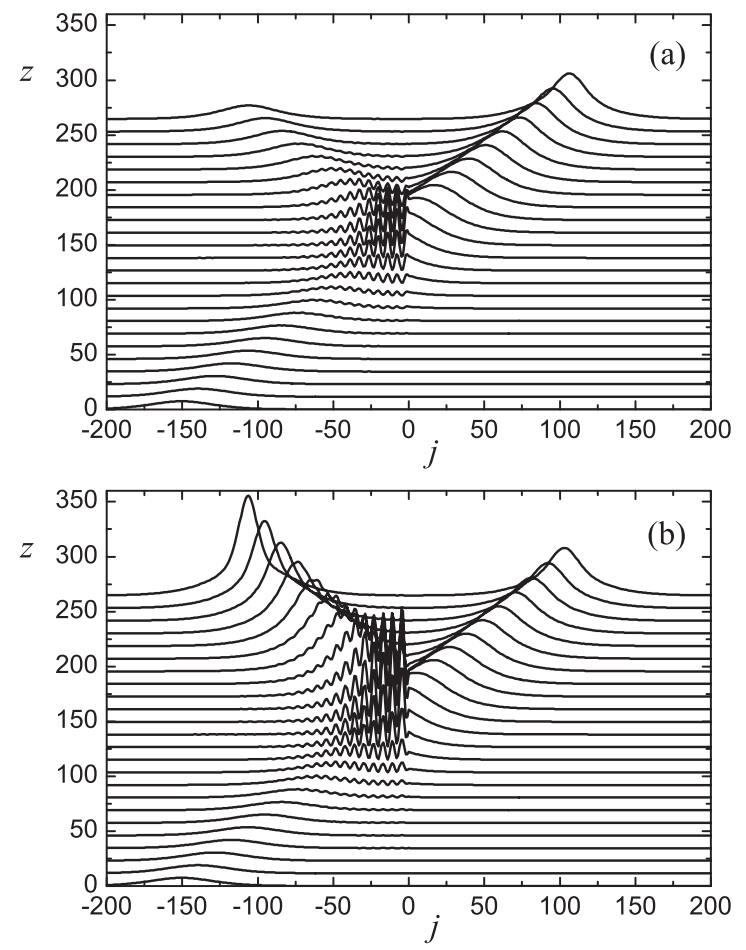

FIG. 5. (a) Example of the soliton scattering for the model parameters $C_{1}=1, C_{2}=4, \rho=-3.7108, \gamma=1\left(\bar{C}_{1}=1 / 4, \bar{\rho} / \bar{\rho}_{\text {crit }}=\right.$ -0.9) and the incident soliton parameters $A=0.05, v=k=0.5$, $j_{0}=-150$. The 3D plot presents $\left|a_{j}(z)\right|^{2}$. Both reflected and transmitted solitons are considerably amplified. (b) Same as in (a) but for the opposite sign of $\rho$.
In Fig. 5(a), we show an example of soliton scattering for model parameters $C_{1}=1, C_{2}=4, \rho=-3.7108, \gamma=1$ and incident soliton parameters $A=0.05, v=k=0.5, j_{0}=$ -150 . The 3D plot presents $\left|a_{j}(z)\right|^{2}$. Both reflected and transmitted solitons are amplified. For chosen model parameters we find $\bar{C}_{1}=1 / 4, \bar{\rho} / \bar{\rho}_{\text {crit }}=-0.9$, and this corresponds to the linear wave scattering presented in Fig. 2(c, left column), where for $k=0.5$ one has $\hat{N}_{T}=|T|^{2}=4.145$ and $\hat{N}_{R}=$ $|R|^{2}=1.535$. This should be compared with the numerically found intensities of transmitted and reflected solitons, $N_{T}=$ $4.169, N_{R}=1.542$. One can see that linear theory gives a very good prediction for scattering parameters of solitons in the regime of weak nonlinearity.

In Fig. 5(b) we show the same as in (a), but for $\rho$ having the opposite sign. Comparison of the results in (a) and (b) confirms the prediction of linear theory that the transmitted waves do not depend on the sign of $\rho$, while the reflected waves do. Transmitted and reflected solitons bare the soliton internal mode.

In Fig. 6, we plot numerical results for the relative soliton scattering intensities $N_{R}$ (white dots) and $N_{T}$ (black dots) given by Eq. (14), as the functions of $\bar{\rho} / \bar{\rho}_{\text {crit }}$. We also plot the analytical result for the linear wave scattering, $\hat{N}_{R}$ (thin line) and $\hat{N}_{T}$ (thick line), given by Eq. (15). Simulation parameters are provided in the figure caption. A remarkably good prediction of the amplitudes of transmitted and reflected solitons is given by the linear theory in the entire range of $\rho$, except for $\left|\bar{\rho} / \bar{\rho}_{\text {crit }}\right|$ close to unity in (a) and (b), where the soliton amplitudes diverge and linear theory becomes invalid.

It is expected that the results of the soliton scattering should deviate from the prediction of linear theory with increasing incident soliton amplitude, $A$. Such deviation is shown in Fig. 7, where we plot $N_{R}$ (white dots), $N_{T}$ (black dots), $\hat{N}_{R}$ (thin lines), and $\hat{N}_{T}$ (thick lines) as the functions of the soliton amplitude $A$. Simulation parameters are listed in the figure
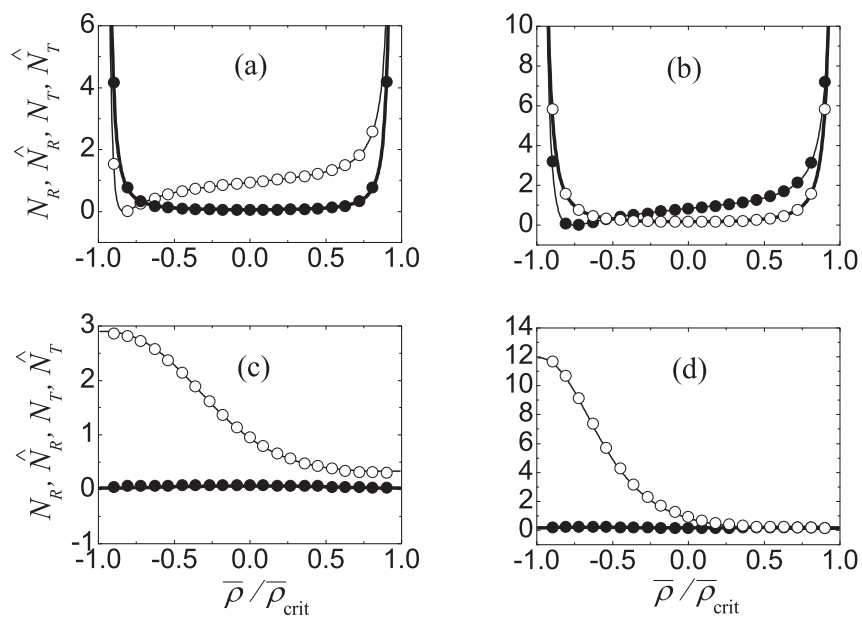

FIG. 6. Numerical results for the soliton scattering, $N_{R}$ (white dots) and $N_{T}$ (black dots), and analytical result for the linear wave scattering, $\hat{N}_{R}$ (thin line) and $\hat{N}_{T}$ (thick line), as the functions of $\bar{\rho} / \bar{\rho}_{\text {crit }}$. Parameters are (a) $C_{1}=1, C_{2}=4$, and $v=k=0.5$; (b) same as in (a) but for $v=k=1$; (c) $C_{1}=4, C_{2}=1$, and $v=k=0.5$; (d) same as in (c) but $v=k=1$. In all cases, for soliton scattering, $\gamma=1$ and incident soliton amplitude $A=0.05$. For linear wave scattering $\gamma=0$. 

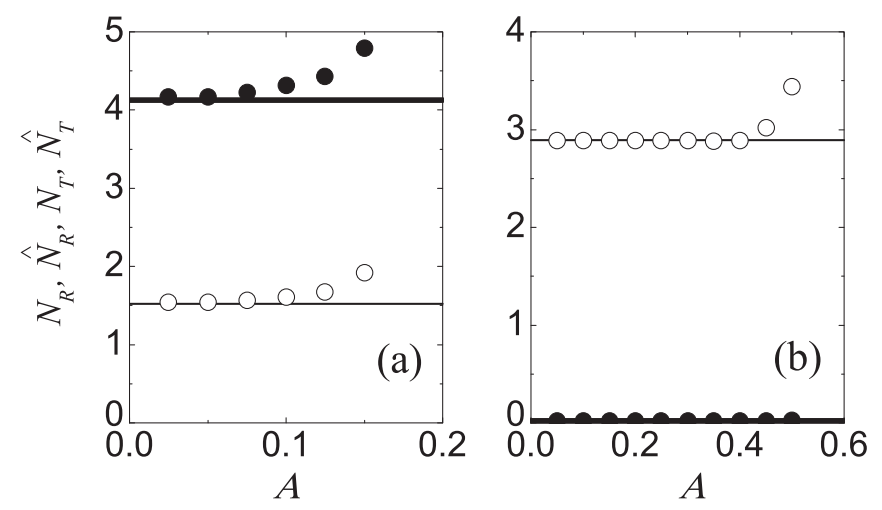

FIG. 7. Numerical results for the soliton scattering, $N_{R}$ (white dots) and $N_{T}$ (black dots), and analytical results for the linear wave scattering, $\hat{N}_{R}$ (thin line) and $\hat{N}_{T}$ (thick line), as the functions of the soliton amplitude $A$. Parameters are (a) $C_{1}=1, C_{2}=4$; (b) $C_{1}=4$, $C_{2}=1$. In all cases, $v=k=0.5, \bar{\rho} / \bar{\rho}_{\text {crit }}=-0.9$. For the soliton scattering $\gamma=1$, and for the linear wave scattering $\gamma=0$.

caption. In (a), both transmitted and reflected solitons are amplified and linear theory gives a good result for $A<0.1$. In (b), the amplitude of the transmitted soliton is nearly zero and only reflected soliton is amplified. In this situation, linear theory works in a wider range of incident soliton amplitude, $A<0.4$.

In all studied cases, fraction of the total power captured by the defect is practically zero. This is expected for the case $\bar{C}_{1}>1$, when no defect modes exist. On the other hand, in the case $\bar{C}_{1}<1$, two such modes do exist [see Eqs. (9) and (10)], but they are not excited in the soliton scattering process. One can check that for $\bar{C}_{1}=1 / 2$ the smallest positive $q$ is achieved when $\rho=C_{2}-C_{1}$ and it is equal to $\sqrt{3}$. This means that the linear nonstaggered localized mode is highly localized and a relatively wide soliton cannot excite it. Nevertheless we expect that specially excited defect modes, using approaches suggested for conservative structures [19,21], may offer extra flexibility to tune the soliton scattering.

\section{CONCLUSIONS}

We have proposed a discrete nonlinear model describing the propagation of electromagnetic waves in a waveguide array with a two-site defect created by a pair of $\mathcal{P} \mathcal{T}$-symmetric waveguides with balanced gain and loss.

In the case of long arrays (when the effects of boundaries are neglected), we have found the profiles of spatially localized defect modes, and also derived the exact relations for the transmission and reflection coefficients of linear waves scattered at the $\mathcal{P} \mathcal{T}$-symmetric defect. For a nonlinear array, we have studied numerically the soliton scattering in the regime of weak nonlinearity, when no excitation of the defect mode is observed.

We have found that the reflected and transmitted linear and nonlinear waves can be substantially amplified by the $\mathcal{P} \mathcal{T}$-symmetric defect, and we have demonstrated that the results of the linear theory give a good prediction for the scattering of relatively wide solitons in the regime of weak nonlinearity.

Our results demonstrate that a pair of $\mathcal{P} \mathcal{T}$-symmetric waveguides with balanced gain and loss can provide a flexible and active control of the propagation of optical beams in waveguide arrays.

\section{ACKNOWLEDGMENTS}

S.V.D. thanks the Nonlinear Physics Center at the Australian National University for warm hospitality during his short visit. This work was partially supported by the Australian Research Council.
[1] Y. J. Chen, A. W. Snyder, and D. N. Payne, IEEE J. Quantum Electron. 28, 239 (1992).

[2] A. Ruschhaupt, F. Delgado, and J. G. Muga, J. Phys. A 38, L171 (2005).

[3] R. El-Ganainy, K. G. Makris, D. N. Christodoulides, and Z. H. Musslimani, Opt. Lett. 32, 2632 (2007).

[4] S. Klaiman, U. Gunther, and N. Moiseyev, Phys. Rev. Lett. 101, 080402 (2008).

[5] K. G. Makris, R. El-Ganainy, D. N. Christodoulides, and Z. H. Musslimani, Phys. Rev. Lett. 100, 103904 (2008).

[6] M. V. Berry, J. Phys. A 41, 244007 (2008).

[7] S. Longhi, Phys. Rev. Lett. 103, 123601 (2009).

[8] S. Longhi, Phys. Rev. A 81, 022102 (2010).

[9] K. G. Makris, R. El-Ganainy, D. N. Christodoulides, and Z. H. Musslimani, Phys. Rev. A 81, 063807 (2010).

[10] M. C. Zheng, D. N. Christodoulides, R. Fleischmann, and T. Kottos, Phys. Rev. A 82, 010103 (2010).

[11] A. Guo, G. J. Salamo, D. Duchesne, R. Morandotti, M. VolatierRavat, V. Aimez, G. A. Siviloglou, and D. N. Christodoulides, Phys. Rev. Lett. 103, 093902 (2009).
[12] C. E. Ruter, K. G. Makris, R. El-Ganainy, D. N. Christodoulides, M. Segev, and D. Kip, Nature Physics 6, 192 (2010).

[13] K. Y. Zhou, Z. Y. Guo, J. C. Wang, and S. T. Liu, Opt. Lett. 35, 2928 (2010).

[14] Z. H. Musslimani, K. G. Makris, R. El-Ganainy, and D. N. Christodoulides, Phys. Rev. Lett. 100, 030402 (2008).

[15] Z. H. Musslimani, K. G. Makris, R. El-Ganainy, and D. N. Christodoulides, J. Phys. A 41, 244019 (2008).

[16] S. V. Dmitriev, A. A. Sukhorukov, and Yu. S. Kivshar, Opt. Lett. 35, 2976 (2010).

[17] H. Ramezani, T. Kottos, R. El Ganainy, and D. N. Christodoulides, Phys. Rev. A 82, 043803 (2010).

[18] A. A. Sukhorukov, Z. Xu, and Yu. S. Kivshar, Phys. Rev. A 82, 043818 (2010).

[19] F. Lederer, G. I. Stegeman, D. N. Christodoulides, G. Assanto, M. Segev, and Y. Silberberg, Phys. Rep. 463, 1 (2008).

[20] A. V. Yulin, D. V. Skryabin, and P. S. J. Russel, Phys. Rev. Lett. 91, 260402 (2003).

[21] W. Krolikowski and Yu. S. Kivshar, J. Opt. Soc. Am. B 13, 876 (1996) 